increase of pulmonary vascular permeability and of lung water, attenuate the sequestration of neutrophils into lungs, alleviate the endotoxemia, decrease the concentrations of THF, IL-8, and PLA2 in plasma, lung tissue, and BALF. Keywords: acute lung injury (ALI); acute respiratory bacteria translocation; distress syndrome (ARDS); endotoxemia; infection, abdominal; lung injury; mechanism; mediators; model; rats; rhubarb; sepsis

Prehosp Disast Med 2002;17(s2):s78-s79.

\section{Computer Simulation for Planning the 3 Ts: Responding to an Airport Disaster} Isao Kamae, $M D, D r P H ;, 1,2$ Hiroki Inoue, $M D, D M D ;{ }^{2}$

Ken Washio, ${ }^{2}$ Shinichiro Yanagisawa, MCS, ${ }^{1}$ Hiroshi Aino, $M S ;^{2}$ Li Cai, $M D ;^{2}$ Ko Nakajo, $M S^{2}$

1. Research Center for Urban Safety and Security, Kobe University, Japan

2. Kobe University School of Medicine, Japan

The purpose of this study was to develop a guideline for the 3 T's: triage, transportation, and treatment in the case of a disaster with large-scale casualties such as a plane crash or explosion at the Kobe Airport. In the aftermath of the Kobe Quake in 1995, Kobe City initiated a new project to build an airport located on the sea next to the artificial Port-Island in the Kobe Bay area. As the airport will be open in 2006, the emergency plan must be developed for medical disaster response at the airport.

All hospitals in Kobe City were evaluated with respect to geographical locations, the availability of transportation from the Kobe Airport, and emergency medicine capability. The information collected was organized to build a network-flow model that logically connects all the hospitals. To analyze the behavior of the model, computer simulation was conducted according to the grades of disaster.

A priority list of the hospitals in terms of the $3 \mathrm{~T}$ 's was constructed corresponding to the grade of disaster. It also indicated the time distributions required for triage and transportation, which suggested the insufficient capability of current hospitals to respond to intermediate or largerscale of disaster.

Computing technology is useful for developing a disaster plan regarding the 3 T's, and has great potential for future improvement combined with geographical information systems.

Keywords: computer simulation; disasters; disaster planning

Prebosp Disast Med 2002;17(s2):s79.

\section{Postmortem Computed Tomography after Unsuccessful Resuscitation of Out-of-Hospital Cardiopulmonary Arrest Patients for Defining Cause of Death}

K. Kuboyama, MD, PhD; S. Marukawa, $M D, P b D ; K$. Yosbibaga, $M D$

Background: It is difficult to definitively diagnose patients with unsuccessfully resuscitated out-of-hospital cardiopulmonary arrest (CPA), because their medical information is extremely limited. It was hypothesized that postmortem computed tomography (CT) scans are helpful in determining their causes of death.

Methods: The records of 50 consecutive, sudden, out-ofhospital CPA patients, who were conveyed to this facility over six months, but who were not resuscitated successfully, were reviewed retrospectively. Medical records, chest and abdominal $x$-ray films, and electrocardiograms were examined if available. CT scans of head, chest, and abdomen were performed after the declaration of death.

Results: Six patients were excluded from the study for various reasons. Forty-four patients were examined using CT (age: $70.5 \pm 11.2$ years, 32 males and 12 females). Head CT was performed in 41 patients, chest in 33 , and abdomen in 23. Definitive diagnoses were made by CT in nine patients (20.5\%) including subarachnoid hemorrhage, aortic dissection, ruptured aortic aneurysm, or severe pneumonia. The suspected diagnosis of acute myocardial infarction for 12 patients $(27.3 \%)$ was supported by calcifications in their coronary arteries. Three patients $(6.8 \%)$ demonstrated cardiac tamponade, but its origin was not defined, whether ruptured aortic aneurysm or a ruptured cardiac ventricle by myocardial infarction. Sixteen patients (36.4\%) were not diagnosed by CT, but six of them had limited information due to the absence of thoracic and abdominal CT.

Conclusion: Postmortem CT scan is informative in defining or suspecting the cause of death in unsuccessfully resuscitated, sudden, out-of-hospital CPA patients.

Keywords: cardiac arrest; etiology; computerized tomography; out-of-hospital; post-mortem

Prebosp Disast Med 2002;17(s2):s79.

\section{Military Unit of the Hospital}

B. Lederman, MD; G. Sadovsky, MD; B. Davidson, $M D$ Assaf Harofeh Medical Center, Israel

Israel presently is preparing itself for the event of chemical warfare. Until 1991, decontamination teams were manned by pre-military youth, ages 16 to 18 years. During the Gulf War, due to the difficulty of the task, it was realized that the role must be filled by adults, and therefore soldiers were designated for these missions. In 1994, special military units were allocated, in order to serve as a contingency unit in each general hospital. The Assaf Harofeh military unit was formed in 1996.

Our hospital is prepared to treat 300 chemical warfare casualties in a mass casualty event. The military unit is made up of around 160 soldiers, as an integral unit of the hospital staff. The unit performs its roles specifically in the contaminated area, side-by-side with the hospital's civilian personnel, under the overall authority of the hospital directors. The roles of this unit are as follows:

1. Evacuation of casualties from the ambulances

2. Undressing and decontamination

3. Ventilating severe casualties

4. Stretcher bearers

5. Transfer of casualties to decontaminated area

The military commander of the unit acts under the direction of the Home Front Command, but receives professional orders and supervision from the hospital administration. Every year, the unit is trained in the hospital, and once every three years participates in an integrative drill simulating a chemical warfare scenario.

Conclusion: Without the integration of the military unit, the hospital would not be prepared for chemical warfare and would be unable to fulfill its goal of saving lives. 